\title{
Key-Rate Bound of a Semi-Quantum Protocol Using an Entropic Uncertainty Relation
}

\author{
Walter O. Krawec \\ Computer Science \& Engineering Department \\ University of Connecticut \\ Storrs, CT 06268 \\ Email: walter.krawec@uconn.edu
}

\begin{abstract}
In this paper we present a new proof technique for semi-quantum key distribution protocols which makes use of a quantum entropic uncertainty relation to bound an adversary's information. Our new technique provides a more optimistic keyrate bound than previous work relying only on noise statistics (as opposed to using additional mismatched measurements which increase the noise tolerance of this protocol, but at the cost of requiring four times the amount of measurement data). Our new technique may hold application in the proof of security of other semi-quantum protocols or protocols relying on two-way quantum communication.
\end{abstract}

\section{INTRODUCTION}

Quantum Key Distribution (QKD) protocols allow for the establishment of a secret key between two parties Alice $(A)$ and $\operatorname{Bob}(B)$ which is secure even against an all-powerful adversary Eve $(E)$ - that is, an adversary bounded only by the laws of physics and not by any computational assumptions as is required when using classical communication only. Numerous QKD protocols have been proposed, many with rigorous proofs of unconditional security. The reader is referred to [1] for a general survey.

However, these QKD protocols, and their security analysis, require both $A$ and $B$ to be "quantum capable." Namely, both $A$ and $B$ must be equipped with devices capable of manipulating quantum resources in certain, arbitrary, ways (e.g., preparing and measuring qubits in certain bases). In 2007, Boyer et al., in [2] introduced the semi-quantum model of cryptography whereby only $A$ was required to be quantum while $B$ was allowed to be very limited and "classical" in his capabilities. These semi-quantum key distribution (SQKD) protocols are interesting to study theoretically as they attempt to answer the question " how quantum does a protocol need to be to gain an advantage over its classical counterpart?" There are also potential practical benefits to studying these protocols: for example, $B$ 's device could be cheaper to manufacture; alternatively, one can consider designing a QKD infrastructure more robust to technical failure - indeed, if a device ever breaks down, one may switch to a "semi-quantum" mode and continue secure operations until the device is fully repaired.

SQKD protocols, however, require a two-way quantum communication channel (one which allows $A$ to send qubits to $B$ who then sends qubits back to $A$ ) greatly increasing the complexity of their security analysis. Though several SQKD protocols have been proposed (see [2], [3], [4], [5], [6], [7],
[8], [9], [10], [11], [12] for a few), until 2015, most were proven only to be robust - a notion introduced in [2] which says any attack which causes $E$ to gain non-zero information on the key must induce a disturbance which can be detected with non-zero probability. Some authors considered security against individual attacks [13] (attacks whereby $E$ is forced to measure her quantum ancilla before the protocol concludes). It wasn't until 2015, that rigorous proofs of security became available in [14], [15], [16].

In a recent work [17], we showed that the original SQKD protocol of Boyer et al., has a noise tolerance of $11 \%$ - exactly the same as the fully-quantum BB84 protocol. Our result in [17], however, required the use of numerous measurements, including mismatched measurements [18], [19]. Ultimately, to compute the key-rate of the Boyer et al., protocol, using our technique in that paper, one must look at over 12 different measurement statistics and then evaluate a series of lengthy equations. (Indeed, our key-rate equation for the SQKD protocol spanned numerous pages!)

In this work, we revisit this semi-quantum protocol and derive a simpler, and far more elegant (in the author's opinion) proof of security using a quantum uncertainty bound to evaluate the von Neumann entropy of the resulting quantum system. Our new bound does not use mismatched measurements (only error rates) and, so, the noise tolerance is not as high as in [17]; however our new result is higher than previous best-known results for this protocol without mismatched measurements. Furthermore, the technique we present here may be simpler to adapt to other SQKD protocols than the technique using mismatched measurements - especially for higher-dimensional protocols (such as [20]) where the technique of mismatched measurements can become intractable.

There are several contributions made in this work, many of which we expect would hold great application outside the scope of this paper. First, we show that for any semiquantum protocol, it is sufficient to consider a restricted form of collective attack. Second, we show an entirely new approach to proving security of semi-quantum protocols; we show how to convert a particular SQKD protocol into an equivalent entanglement based version and we derive a new key-rate bound which does not require the use of numerous mismatched measurement statistics and which produces a higher noise tolerance than previous work without these statistics (along 
with a far simpler key-rate expression). Note that, in [21], a technique of converting certain two-way QKD protocols into equivalent entanglement based versions was shown; however their result could only be applied to protocols where $B$ 's output is independent of his input averaged over all of his operations - this property is sadly lacking in the semi-quantum model and so a new method is required which we introduce in this paper. Third, our proof shows a new and interesting application of a quantum uncertainty bound to the semi-quantum model of cryptography and also an interesting application of a continuity bound on conditional von Neumann entropy which may be of great use when proving security of new protocols in the semi-quantum model (or, more generally, for protocols relying on a two-way channel which may not hold certain symmetry properties).

\section{A. Notation}

We assume the reader is familiar with basic quantum information theory and so here we will only introduce our notation and a few general concepts; for a general survey see [22]. The computational $Z$ basis is defined to be $\{|0\rangle,|1\rangle\}$ while the Hadamard $X$ basis is $\{|+\rangle,|-\rangle\}$, where $| \pm\rangle=\frac{1}{\sqrt{2}}(|0\rangle \pm|1\rangle)$.

We denote by $H\left(p_{1}, \cdots, p_{n}\right)$ to be the Shannon entropy of $p_{1}, \cdots, p_{n}$. If $A$ and $B$ are random variables, then $H(A \mid B)$ is the conditional Shannon entropy of $A$ conditioned on $B$. By $h(x)$ we mean the binary entropy function: $h(x)=H(x, 1-$ $x)=-x \log x-(1-x) \log (1-x)$. All logarithms in this paper are base two.

A density operator is a Hermitian positive semi-definite operator of unit trace. If $\rho$ is a density operator acting on Hilbert space $\mathcal{H}_{A} \otimes \mathcal{H}_{B}$, we often write $\rho_{A B}$. In this case, we write $\rho_{B}$ to mean the operator resulting from tracing out $A$; i.e., $\rho_{B}=\operatorname{tr}_{A} \rho_{A B}$. Similarly when the operator acts on larger systems. We also will write $\mathcal{H}_{A B}$ to denote $\mathcal{H}_{A} \otimes \mathcal{H}_{B}$.

Given density operator $\rho_{A B}$ we write $S(A B)_{\rho}$ to mean the von Neumann entropy of $\rho_{A B}$. We write $S(A \mid B)_{\rho}$ to mean the conditional von Neumann entropy: $S(A \mid B)_{\rho}=$ $S(A B)_{\rho}-S(B)_{\rho}$. If the context is clear, we will forgo writing the subscript " $\rho$."

Given an operator $A$, we write $\|A\|$ to mean the trace norm of $A$. If $A$ is Hermitian and finite dimensional, then $\|A\|=$ $\sum_{i}\left|\lambda_{i}\right|$, where $\left\{\lambda_{i}\right\}$ are the eigenvalues of $A$.

If $z \in \mathbb{C}^{m \times n}$, then we write $z^{*}$ to mean the conjugate transpose of $z$. Also, we define $\mathbb{D}=\{z \in \mathbb{C}|| z \mid \leq 1\}$. Finally, we write $[\mathbf{a}, \mathbf{b}]_{A B}$ to mean $|a, b\rangle\left\langle a,\left.b\right|_{A B}\right.$.

\section{B. (S)QKD Security}

A $(\mathrm{S}) \mathrm{QKD}$ protocol operates in two stages: first a quantum communication stage whereby users utilize the communication resources available to them (typically a quantum channel and an authenticated classical channel) to establish a raw-key which is a string of 0's and 1's which is partially correlated and partially secret. In general, this yields a classical-classicalquantum state of the form:

$$
\rho_{A B E}=\sum_{a, b \in\{0,1\}^{n}}[\mathbf{a}, \mathbf{b}]_{A B} \otimes \rho_{E}^{(a, b)},
$$

where the $A$ and $B$ register represent $A$ and $B$ 's raw-key respectively, while $\rho_{E}^{(a, b)}$ (which is not necessarily of unit trace) represents the state of $E$ 's memory in the event $A$ and $B$ have a raw-key of $a$ and $b$ respectively. Following the quantum communication stage, a classical stage consisting of error correction and privacy amplification is run producing a secret key of size $\ell(n)$ bits which may then be used for other cryptographic protocols.

An important question is, given certain observations on $E$ 's attack (e.g., the error rate), how large is $\ell(n)$ ? For collective attacks (attacks whereby $E$ performs the same attack operation each iteration however is free to postpone measurement of her ancilla until any future time of her choice), Equation 1 takes on the simpler form:

$$
\rho_{A B E}=\left(\sum_{a, b \in\{0,1\}}[\mathbf{a}, \mathbf{b}]_{A B} \otimes \rho_{E}^{(a, b)}\right)^{\otimes n},
$$

in which case the Devetak-Winter key-rate expression [23] may be employed which states:

$$
r:=\lim _{n \rightarrow \infty} \frac{\ell(n)}{n}=\inf [S(A \mid E)-H(A \mid B)],
$$

where the infimum is over all collective attacks which induce the observed noise statistics. Computing a bound on this expression is the key critical element of any (S)QKD security proof [1].

\section{THE PROTOCOL}

The protocol we consider is the original SQKD protocol of Boyer et al., introduced in [2], [3]. This protocol, being a semi-quantum one, assumes that $A$ is fully quantum in that she can prepare and measure qubits in arbitrary bases; however $B$ is classical in that he can only directly work with the computational $Z$ basis. In more detail, a SQKD protocol utilizes a two-way quantum channel. We call the channel connecting $A$ to $B$ the forward channel and the channel connecting $B$ to $A$ the reverse channel. Each iteration of the quantum communication stage, $A$ will prepare and send a qubit. $B$ is then restricted to choosing between two operations: Measure and Resend or to Reflect. If he chooses Measure and Resend, he will subject the incoming qubit to a $Z$ basis measurement and prepare a new qubit in the same state he observed (i.e., if his measurement result is $|r\rangle$, for $r \in\{0,1\}$, he will send a qubit $|r\rangle$ back to $A$ ); if he chooses Reflect he will completely disconnect from the quantum channel, allowing the qubit to pass through his lab undisturbed, and return to $A$ (in essence, if $B$ chooses Reflect, $A$ is "talking to herself").

The protocol we consider, and which we denote as $\Pi^{\mathrm{SQKD}}$, operates as follows:

1) $A$ chooses to send a qubit of the form $|0\rangle,|1\rangle,|+\rangle$, or $|-\rangle$, choosing randomly.

2) $B$ will choose to Measure and Resend or to Reflect. If he chooses Measure and Resend he 
will save his measurement result in a classical register to serve as his potential raw-key bit for this iteration.

3) $A$ will measure in the same basis she originally used to send.

4) $A$ will disclose her choice of basis; $B$ will disclose his choice of operation (Measure and Resend or Reflect). This is done using an authenticated classical channel.

5) If $A$ used the $Z$ basis and if $B$ chose to Measure and Resend, then they will use this iteration to contribute towards their raw-key. $A$ will use her initial preparation choice as her key bit (equivalently, she may use her measurement result at the end - our security analysis will apply to both cases). Other iterations, along with a suitably sized random subset of these "raw-key" iterations, may be used to estimate the error rate in the channel. In particular, $A$ and $B$ may estimate the $Z$ basis error rate in the forward, reverse, and joint channel. They may also observe the $X$ basis error rate in the joint channel (but not the forward or reverse separately since $B$ is unable to prepare or measure in the $X$ basis).

It is not difficult to see that the protocol is correct. We analyze its security by determining a new lower-bound on the Devetak-Winter key-rate expression (Equation 2) for this protocol.

\section{SECURITY PROOF}

We prove security against collective attacks in this paper we will comment on general attacks later. Collective attacks are those where $E$ applies the same attack operation each iteration of the protocol, but is free to postpone measurement of her ancilla until any future time of her choice. In the semiquantum model, where an attacker has two opportunities to attack a qubit each iteration, a collective attack is a pair of unitary operators $\left(U_{F}, U_{R}\right)$, each acting on the Hilbert space $\mathcal{H}_{T} \otimes \mathcal{H}_{E}$ (here $\mathcal{H}_{T}$ is the two-dimensional space modeling the qubit "transit" space while $\mathcal{H}_{E}$ is $E$ 's quantum ancilla). The operator $U_{F}$ is applied in the Forward channel (as a qubit travels from $A$ to $B$ ) while $U_{R}$ is applied in the Reverse channel (when the qubit returns from $B$ to $A$ ).

Our proof of security follows three steps. First, we will prove that for any semi-quantum protocol, it is sufficient to consider a particular "restricted" collective attack which is easier to analyze mathematically, but does not cause $E$ to lose attack power. Second, using this result, we show how to convert the protocol of interest into a mathematically equivalent entanglement based version. Third, we use a quantum uncertainty bound and a continuity bound on conditional entropy to analyze the entanglement based version - security of the SQKD protocol will then follow. See Figure 1 .

\section{A. Restricted Attacks}

In [5], we showed that for any single-state SQKD protocol (i.e., those where $A$ is restricted to sending a single, publicly known, qubit state each iteration, typically $|+\rangle[4]$ ), to prove security against collective attacks it is sufficient to consider

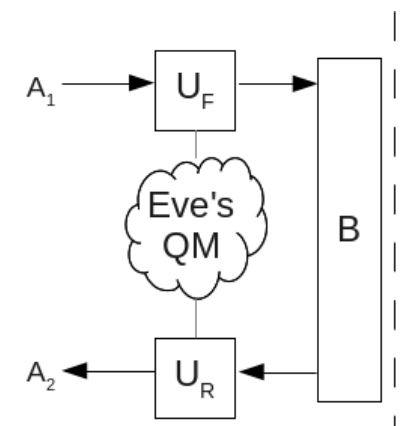

(1)

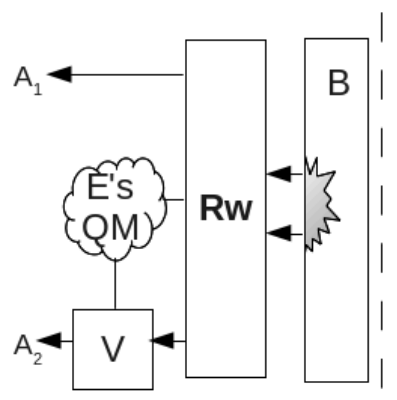

(3)

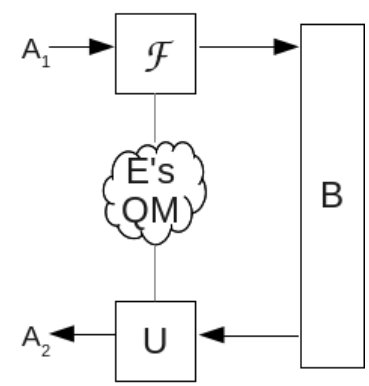

(2)

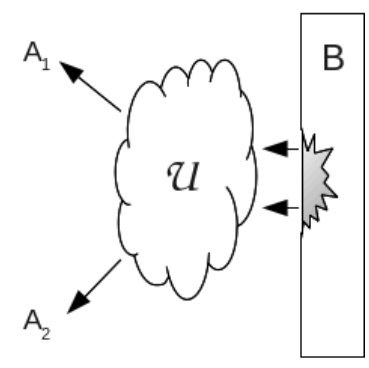

(4)
Fig. 1. Our security proof in pictures (note: $Q M=$ "Quantum Memory"). (1) is an arbitrary collective attack against the actual SQKD protocol (with classical Bob) that we wish to analyze. We show that for every such attack, there is an equivalent restricted attack (2). Next, we show that, mathematically, analyzing (2) is the same as analyzing a different protocol where a quantum Bob prepares pairs of qubits (and his qubit source is partially influenced by Eve, represented here as a shaded region in $B$ 's lab) and sends them to Alice; namely, for every restricted attack against the SQKD protocol, there is an equivalent attack against our new protocol where first Eve "rewinds" the channel state with operator Rw (simulating the system had $A$ sent a qubit first instead of $B$ ), and then attacks one of the qubits. Of course analyzing (4), where Eve is not restricted to performing this "rewind" attack, only gives her more power. Thus security in (4) $\Rightarrow(3) \Rightarrow(2) \Rightarrow(1)$, our original goal.

security against a "restricted" attack whereby Eve, in the forward channel, need only bias Bob's measurement result; in the reverse channel, she applies an arbitrary unitary operator. That is, it is not required that she perform an arbitrary unitary operator in the forward channel, entangling the qubit with her private quantum memory. Such attacks are easier to analyze and have been used in [15], [16] to show security of several different single-state protocols - however, as shown in [5], the result is only correct for single state protocols. The original SQKD protocol of Boyer et al., which we are considering in this work (i.e., $\Pi^{\mathrm{SQKD}}$ ), is a multi-state protocol, one where $A$ prepares different states each iteration, choosing randomly each time. However, it remained an open question as to whether or not some other form of restricted attack might be constructed for multi-state protocols. We answer this question in the affirmative.

Definition III.1. Let $\mathcal{B}=\left\{\left|v_{0}\right\rangle,\left|v_{1}\right\rangle\right\}$ be an orthonormal basis. A multi-state restricted collective attack with respect to $\mathcal{B}$ is a tuple $\left(q_{0}, q_{1}, \eta_{0}, \eta_{1}, U\right)$, where $q_{0}, q_{1} \in[0,1] ; \eta_{0}, \eta_{1} \in \mathbb{D}$ subject to the restriction that $q_{0} \eta_{1} \sqrt{1-q_{1}^{2}}+q_{1} \eta_{0}^{*} \sqrt{1-q_{0}^{2}}=$ 0 ; and $U$ is a unitary operator acting on $\mathcal{H}_{T} \otimes \mathcal{H}_{E}$. The attack 
consists of the following actions:

1) When $E$ first captures the qubit from $A$ in the forward channel, she applies the operator $\mathcal{F}$, acting on $\mathcal{H}_{T} \otimes \mathcal{H}_{E}$ which acts as follows:

$$
\begin{aligned}
& \mathcal{F}\left|v_{0}\right\rangle=q_{0}|0,0\rangle_{T E}+\sqrt{1-q_{0}^{2}}|1, e\rangle_{T E} \\
& \mathcal{F}\left|v_{1}\right\rangle=\sqrt{1-q_{1}^{2}}|0, f\rangle_{T E}+q_{1}|1,0\rangle_{T E},
\end{aligned}
$$

where:

$$
\begin{aligned}
& |e\rangle=\eta_{0}|0\rangle_{E}+\sqrt{1-\left|\eta_{0}\right|^{2}}|1\rangle_{E} \\
& |f\rangle=\eta_{1}|0\rangle_{E}+\sqrt{1-\left|\eta_{1}\right|^{2}}|1\rangle_{E} .
\end{aligned}
$$

Note that it is not difficult to see, considering the restrictions on the values $\eta_{0}, \eta_{1}, q_{0}$, and $q_{1}$, that $\mathcal{F}$ is an isometry and may therefore be easily extended to a unitary operator; thus this is an operation $E$ can perform within the laws of quantum physics.

2) When the qubit returns from $B$ (on its way back to $A$ in the reverse channel), $E$ captures it again, and applies the unitary operator $U$.

When the context is clear, we will simply call the above attack a restricted attack as opposed to its longer title. The following theorem proves that it is sufficient to consider these restricted attacks when proving security of any semi-quantum protocol against arbitrary collective attacks.

Theorem 1. Let $\mathcal{B}=\left\{\left|v_{0}\right\rangle,\left|v_{1}\right\rangle\right\}$ be an arbitrary orthonormal basis. For every collective attack $\mathcal{C}=\left(U_{F}, U_{R}\right)$, there exists a restricted attack $\mathcal{R}=\left(q_{0}, q_{1}, \eta_{0}, \eta_{1}, U\right)$ such that, for any SQKD protocol with quantum $A$ and classical $B$, the following are true:

1) $A$ and $B$ cannot distinguish between attack $\mathcal{C}$ and $\mathcal{R}$.

2) $E$ 's final quantum system is the same regardless of whether she used $\mathcal{C}$ or $\mathcal{R}$.

3) The key-rate is equal under both attacks.

Proof. Let $\mathcal{B}$ be given and fix a collective attack $\mathcal{C}=$ $\left(U_{F}, U_{R}\right)$, where $U_{F}$ is a unitary operator applied in the forward channel while $U_{R}$ is a unitary operator applied in the reverse. We will construct a restricted attack $\mathcal{R}$ satisfying the required conditions. Without loss of generality, we may assume E's system is cleared to some "zero" state $|\chi\rangle_{E}$ at the start of the attack. In this case, we may write $U_{F}$ 's action on basis states as follows:

$$
\begin{aligned}
& U_{F}\left|v_{0}, \chi\right\rangle=\alpha\left|0, e_{0}\right\rangle_{T E}+\sqrt{1-\alpha^{2}}\left|1, e_{1}\right\rangle_{T E} \\
& U_{F}\left|v_{1}, \chi\right\rangle=\sqrt{1-\beta^{2}}\left|0, e_{2}\right\rangle_{T E}+\beta\left|1, e_{3}\right\rangle_{T E}
\end{aligned}
$$

where $\alpha, \beta \in[0,1]$ (any phase may be absorbed into the vectors $\left|e_{i}\right\rangle$ ) and the $\left|e_{i}\right\rangle$ are arbitrary normalized (though not necessarily orthogonal) states in $\mathcal{H}_{E}$. Let $q_{0}=\alpha$ and $q_{1}=\beta$ for our restricted attack.

Unitarity of $U_{F}$ imposes the following condition:

$$
\alpha \sqrt{1-\beta^{2}}\left\langle e_{0} \mid e_{2}\right\rangle+\beta \sqrt{1-\alpha^{2}}\left\langle e_{1} \mid e_{3}\right\rangle=0 .
$$

Let $\eta_{0}=\left\langle e_{3} \mid e_{1}\right\rangle$ and $\eta_{1}=\left\langle e_{0} \mid e_{2}\right\rangle$ for our restricted attack $\mathcal{R}$ (this clearly satisfies the required restrictions on $q_{i}$ and $\eta_{i}$ ). Now, first consider the case that $\left|\eta_{i}\right|<1$ (the case when $\left|\eta_{i}\right|=1$ for one or both $i$ will be considered afterwards). Let $V$ be the operator acting on states in $\mathcal{H}_{T} \otimes \mathcal{H}_{E}$ as follows:

$$
\begin{array}{ll}
V|0,0\rangle_{T E}=\left|0, e_{0}\right\rangle & V|1,0\rangle_{T E}=\left|1, e_{3}\right\rangle \\
V|0,1\rangle_{T E}=\left|0, g_{0}\right\rangle & V|1,1\rangle_{T E}=\left|1, g_{1}\right\rangle
\end{array}
$$

where:

$$
\left|g_{0}\right\rangle=\frac{\left|e_{2}\right\rangle-\eta_{1}\left|e_{0}\right\rangle}{\sqrt{1-\left|\eta_{1}\right|^{2}}}, \quad\left|g_{1}\right\rangle=\frac{\left|e_{1}\right\rangle-\eta_{0}\left|e_{3}\right\rangle}{\sqrt{1-\left|\eta_{0}\right|^{2}}}
$$

We claim that $V$ is an isometry. First, it is clear that each $\left|g_{i}\right\rangle$ is normalized. Indeed:

$$
\begin{aligned}
\left\langle g_{0} \mid g_{0}\right\rangle & =\frac{1+\left|\eta_{1}\right|^{2}-2 \operatorname{Re}\left(\eta_{1}\left\langle e_{2} \mid e_{0}\right\rangle\right)}{1-\left|\eta_{1}\right|^{2}} \\
& =\frac{1+\left|\eta_{1}\right|^{2}-2 \operatorname{Re}\left|\eta_{1}\right|^{2}}{1-\left|\eta_{1}\right|^{2}}=1,
\end{aligned}
$$

where, above, we used the fact that $\eta_{1}=\left\langle e_{0} \mid e_{2}\right\rangle$. A similar computation yields $\left\langle g_{1} \mid g_{1}\right\rangle=1$. What remains to be shown is that $\left\langle e_{0} \mid g_{0}\right\rangle=\left\langle e_{3} \mid g_{1}\right\rangle=0$. But this is clear:

$$
\left\langle e_{0} \mid g_{0}\right\rangle=\frac{\left\langle e_{0} \mid e_{2}\right\rangle-\eta_{1}}{\sqrt{1-\left|\eta_{0}\right|^{2}}}=\frac{\eta_{1}-\eta_{1}}{\sqrt{1-\left|\eta_{0}\right|^{2}}}=0,
$$

and similarly for $\left\langle e_{3} \mid g_{1}\right\rangle$. We conclude, therefore, that $V$ is an isometry which may be extended to a unitary operator (its action on states not shown above is irrelevant). In the following, we will assume $V$ is simply unitary.

To simplify notation in the remainder of this proof, let $N_{i}=$ $\sqrt{1-\left|\eta_{i}\right|^{2}}$. By linearity of $V$, it follows that:

$$
\begin{aligned}
V|1, e\rangle_{T E} & =V\left(\eta_{0}|1,0\rangle+N_{0}|1,1\rangle\right) \\
& =\eta_{0}\left|1, e_{3}\right\rangle+N_{0}|1\rangle \otimes\left(\frac{\left|e_{1}\right\rangle-\eta_{0}\left|e_{3}\right\rangle}{N_{0}}\right) \\
& =\left|1, e_{1}\right\rangle,
\end{aligned}
$$

and similarly, $V|0, f\rangle=\left|0, e_{2}\right\rangle$ (where $|e\rangle$ and $|f\rangle$ are the states resulting from the action of the restricted attack operator $\mathcal{F}$ defined in Equations 4 and 5 .

Let $U=U_{R} V$. We claim that $\mathcal{R}=\left(q_{0}, q_{1}, \eta_{0}, \eta_{1}, U\right)$, as constructed above, is the desired restricted attack. (Note, we are still assuming for the time being, that $\left|\eta_{i}\right|<1$.)

We first consider the case where $A$ 's sent state is pure; for mixed states the result will follow immediately due to linearity of the operations. On any particular iteration of the protocol, let $|a\rangle=x\left|v_{0}\right\rangle+y\left|v_{1}\right\rangle$ be the state prepared and sent by $A$. These $x$ and $y$ are potentially known only to $A$ (i.e., $A$ may choose, each iteration, to send a randomly prepared state in which case the $x$ and $y$ are chosen randomly). Let $\left|\psi_{R}\right\rangle$ be the state of the qubit as it arrives to $B$ if $E$ uses the restricted attack as constructed above. Let $\left|\psi_{C}\right\rangle$ be the state if $E$ uses 
the collective attack $\mathcal{C}=\left(U_{F}, U_{R}\right)$. These are both easily computed:

$$
\begin{array}{r}
\left|\psi_{R}\right\rangle=\mathcal{F}|a\rangle=|0\rangle \otimes\left(x q_{0}|0\rangle_{E}+y \sqrt{1-q_{1}^{2}}|f\rangle_{E}\right) \\
+|1\rangle \otimes\left(x \sqrt{1-q_{0}^{2}}|e\rangle_{E}+y q_{1}|0\rangle_{E}\right) \\
\left|\psi_{C}\right\rangle=U_{F}|a, \chi\rangle=|0\rangle \otimes\left(x \alpha\left|e_{0}\right\rangle+y \sqrt{1-\beta^{2}}\left|e_{2}\right\rangle\right) \\
+|1\rangle \otimes\left(x \sqrt{1-\alpha^{2}}\left|e_{1}\right\rangle+y \beta\left|e_{3}\right\rangle\right) .
\end{array}
$$

At this point, $B$ will either Measure and Resend (saving his measurement result in a private register) or Reflect. We first consider the case where he chooses Measure and Resend. Let $\rho^{R}$ be the result of this operation in the restricted attack case and $\rho^{C}$ the result in the general collective attack case. These density operators are found to be:

$$
\begin{aligned}
\rho^{R} & =[\mathbf{0}, \mathbf{0}]_{B T} \otimes P\left(x q_{0}|0\rangle_{E}+y \sqrt{1-q_{1}^{2}}|f\rangle_{E}\right) \\
& +[\mathbf{1}, \mathbf{1}]_{B T} \otimes P\left(x \sqrt{1-q_{0}^{2}}|e\rangle_{E}+y q_{1}|0\rangle_{E}\right) \\
\rho^{C} & =[\mathbf{0}, \mathbf{0}]_{B T} \otimes P\left(x \alpha\left|e_{0}\right\rangle+y \sqrt{1-\beta^{2}}\left|e_{2}\right\rangle\right) \\
& +[\mathbf{1}, \mathbf{1}]_{B T} \otimes P\left(x \sqrt{1-\alpha^{2}}\left|e_{1}\right\rangle+y \beta\left|e_{3}\right\rangle\right)
\end{aligned}
$$

where $P(z)=z z^{*}$. At this point, if $E$ is using the restricted attack, she will now apply the operator $U=U_{R} V$. However, $V$ 's action of $\rho^{R}$ evolves the state to:

$$
\begin{aligned}
V \rho^{R} V^{*} & =[\mathbf{0}, \mathbf{0}]_{B T} \otimes P\left(x \alpha\left|e_{0}\right\rangle+y \sqrt{1-\beta^{2}}\left|e_{2}\right\rangle\right) \\
& +[\mathbf{1}, \mathbf{1}]_{B T} \otimes P\left(x \sqrt{1-\alpha^{2}}\left|e_{1}\right\rangle+y \beta\left|e_{3}\right\rangle\right) \\
& =\rho^{C} .
\end{aligned}
$$

Thus, it is clear that $U \rho^{R} U^{*}=U_{R} \rho^{C} U_{R}^{*}$ and so the resulting quantum state is identical regardless of whether $E$ used the restricted attack $\mathcal{R}$ or the collective attack $\mathcal{C}(A, B$, and $E$ 's systems are identical regardless).

We now consider the case when $B$ reflects. Let $\sigma^{R}$ be the state of the system when the qubit leaves $B$ 's lab and $E$ used the restricted attack; let $\sigma^{C}$ be the state of the system if $E$ uses the collective attack. That is: $\sigma^{R}=\left|\psi_{R}\right\rangle\left\langle\psi_{R}\right|$ and $\sigma^{C}=$ $\left|\psi_{C}\right\rangle\left\langle\psi_{C}\right|$, where $\left|\psi_{R}\right\rangle$ and $\left|\psi_{C}\right\rangle$ are defined in Equations 6 and 7 It is trivial to show that $V \sigma^{R} V^{*}=\sigma^{C}$ and so the result holds in the case $B$ reflects.

In the above, we assumed that $\left|\eta_{i}\right|<1$ for both $i=0,1$. However, if $\left|\eta_{i}\right|=1$ for one or both $i$, then $|1,1\rangle_{T E}$ (if $\left|\eta_{0}\right|=$ 1 ) or $|0,1\rangle_{T E}$ (if $\left|\eta_{1}\right|=1$ ) never appear in the state following $E$ 's application of $\mathcal{F}$. Note also that, if $\left|\eta_{0}\right|=1$ then, it must hold that $\left|e_{3}\right\rangle=e^{i \theta}\left|e_{1}\right\rangle$ for some $\theta$ (and a similar statement may be made if $\left|\eta_{1}\right|=1$ ); this phase change will be done by $\mathcal{F}$ in the forward direction and there is no need to "create" the $\left|e_{1}\right\rangle$ state later in the reverse, only the $\left|e_{3}\right\rangle$ state (if $\left|\eta_{1}\right|=1$, then $\left|e_{2}\right\rangle=e^{i \theta^{\prime}}\left|e_{0}\right\rangle$ and so there is no need to "create" the $\left|e_{2}\right\rangle$ state later, only change the phase which is done by $\mathcal{F}$ ). Thus, $V$ 's action on these states $(|1,1\rangle$ or $|0,1\rangle)$ may be arbitrary and we need not define the corresponding $\left|g_{i}\right\rangle$. It is clear, then, that $V$ may be made a unitary operator, and the rest of the proof follows as above.

We conclude, therefore, that regardless of $A$ or $B$ 's choices, the state of the quantum system for all three parties is the same whether $E$ used $\mathcal{C}$ or $\mathcal{R}$ (meaning the resulting density operator describing the joint systems are equal). Thus the view from $A, B$, and $E$ 's point of view are identical in both cases; furthermore, the key-rate computation (Equation 2) will also be identical in both cases.

Thus, to prove security of any SQKD protocol, it is sufficient to consider only a restricted collective attack - security against general collective attacks will follow from that. In the next section, we will show how this result may be used for a particular protocol to convert it into an equivalent entanglement based version from which a quantum uncertainty bound may be used to compute the key-rate.

Note that the choice of basis $\mathcal{B}$ is irrelevant to the attacker. Thus, when analyzing the security of a SQKD protocol using this result, one is free to choose a basis that simplifies the analysis and computations. In the remainder of this paper, our proof assumes $\mathcal{B}=Z=\{|0\rangle,|1\rangle\}$. Also note that our proof would hold even for protocols where $B$ performs a CNOT gate (acting on $\mathcal{H}_{T}$ and his private register) instead of a projective $Z$ basis measurement when he chooses Measure and Resend; mathematically, the two operations will be identical in this case, and the proof above follows through identically.

Before concluding this section, we point out a simplification of our definition if we assume E's attack is symmetric. This is an assumption often made in quantum security proofs and can even be enforced by the parties. In particular, if the $Z$ basis error induced by $E$ 's attack in the forward channel can be parameterized by a single variable $Q$ (i.e., the probability of an $|i\rangle$ flipping to a $|1-i\rangle$ in the forward channel is $Q$ ), and if we work with respect to the $Z$ basis (i.e., $\left|v_{i}\right\rangle=|i\rangle$ ), then the restricted attack adopts a far simpler form:

Definition III.2. A symmetric restricted collective attack is a tuple $\mathcal{R}_{\text {sym }}=(Q, \eta, U)$, where $Q \in[0,1], \eta \in \mathbb{D}$, and $U$ is a unitary operator acting on $\mathcal{H}_{T} \otimes \mathcal{H}_{E}$. This attack follows the same process as in Definition III.1, setting $\mathcal{R}=\left(\sqrt{1-Q}, \sqrt{1-Q}, \eta,-\eta^{*}, U\right)$.

It is not difficult to see that if $\left(q_{0}, q_{1}, \eta_{0}, \eta_{1}, U\right)$ is a symmetric attack (i.e., $q_{0}=\sqrt{1-Q}=q_{1}$ ), then it must hold that $\eta_{1}=-\eta_{0}^{*}$.

\section{B. An Entanglement Based Protocol}

Our conversion from the prepare-and-measure protocol $\Pi^{\mathrm{SQKD}}$ to an equivalent, entanglement based one, follows several reductions. Our goal in this section is to construct a new protocol whereby $B$ (who is no longer classical) prepares quantum states and sends them to $A$ (who is still quantum). 
However, by analyzing the security of this new protocol, we will show security of the SQKD protocol $\Pi^{\mathrm{SQKD}}$.

First, note that B's Measure and Resend operation may be equivalently modeled as a CNOT gate acting on the qubit and an empty register private to $B$ [3]. Of course, his Reflect operation may be modeled as the identity operation. Thus, when analyzing $\Pi^{\mathrm{SQKD}}$ we may instead analyze the case where $B$ applies a unitary operation acting on the qubit and a register private to him. Second, we may assume that $A$, instead of preparing and sending a random state of the form $|0\rangle,|1\rangle,|+\rangle$, or $|-\rangle$, will instead prepare a Bell state of the form $\frac{1}{\sqrt{2}}(|00\rangle+|11\rangle)$, sending one particle to $B$ while keeping the other particle in her private lab. Standard arguments apply to show that security of this new protocol (which we denote by $\widetilde{\Pi}^{\mathrm{SQKD}}$ ) implies security of the prepare and measure one $\Pi^{\mathrm{SQKD}}$. Furthermore, Theorem 1 still applies (see the comment after the proof). It is clear that, if we prove security of $\widetilde{\Pi}^{\mathrm{SQKD}}$ then security of $\Pi^{\mathrm{S} Q \mathrm{KD}}$ will follow.

Now, consider the following protocol which we denote by $\Pi^{*}$, whose quantum communication stage consists of:

1) $B$ prepares the state $\sqrt{p_{0}}|000\rangle_{A_{1} A_{2} B}+\sqrt{p_{1}}|110\rangle_{A_{1} A_{2} B}$ if he wishes to "Reflect" otherwise he prepares the state $\sqrt{p_{0}}|000\rangle_{A_{1} A_{2} B}+\sqrt{p_{1}}|111\rangle_{A_{1} A_{2} B}$ if he wishes to "Measure and Resend" (he will chose the operation randomly each iteration). The two qubits $A_{1}$ and $A_{2}$ are sent to Alice. Note that the terminology "Measure and Resend" and "Reflect" no longer has any real meaning in this protocol.

2) $A$ receives both particles $A_{1}$ and $A_{2}$ and will measure each in the $Z$ basis or the $X$ basis, choosing randomly.

3) If $B$ chooses to Measure and Resend and if $A$ uses the $Z$ basis, they may use their results as their raw-key bit (we assume $A_{1}$ is used as $A$ 's raw-key bit, though our analysis below would be symmetric if $A_{2}$ were used instead). Other iterations, along with a random subset of these "raw-key" iterations, may be used for error estimation in the obvious way.

We give $E$ the ability to control the setting of $p_{0}$ and $p_{1}$ which can only increase her power (and, as a consequence, also gives us partial device independence - indeed, one can consider the scenario that $E$ manufactured the device $B$ is using and programmed in a particular $p_{0}$ and $p_{1}$ value). A collective attack against this protocol, thus, is a setting for $p_{0}$ (the value $p_{1}=1-p_{0}$ of course) and a unitary operator $U$ acting on two qubits $A_{1}$ and $A_{2}$ and $E$ 's private quantum memory.

While $\Pi^{*}$ is not a "true" entanglement-based version (as $B$ is making a choice between two pure states), it would not be difficult to make it one simply by increasing the dimension of $B$ 's space with an extra qubit (which, after measuring, would determine his choice of Measure and Resend or Reflect). However, as it turns out, the protocol as described will be sufficient to complete our security analysis of the prepare-and-measure protocol.

We claim that, if $\Pi^{*}$ is secure, then so is $\widetilde{\Pi}^{\mathrm{SQKD}}$ (in which case, so is $\left.\Pi^{\mathrm{S} Q K D}\right)$. In particular, we will show that, for any attack against $\widetilde{\Pi}^{\mathrm{SQKD}}$, there exists an attack against $\Pi^{*}$ which exactly replicates the resulting quantum system. In particular, given an attack against $\widetilde{\Pi}^{\mathrm{SQKD}}$, we will construct an attack against $\Pi^{*}$ which first "rewinds" the forward channel attack simulating the system had $A$ initially sent a qubit as opposed to $B$. Informally, as an example, if $B$ sends a $|0\rangle$ in the $A_{1}$ register (which he never does - it is always a pair of qubits but this is simply for illustration), then we construct an attack which causes $A$ to receive a $|0\rangle$ or $|1\rangle$ in her $A_{1}$ register with the same probabilities as if she had sent a $|0\rangle$ or a $|1\rangle$ and $B$ happened to measure a $|0\rangle$ if they were running $\widetilde{\Pi}^{\mathrm{SQKD}}$. Furthermore, E's memory will be in the same state in both cases. Thus, we will "rewind" the forward channel for $\Pi^{*}$ to simulate the scenario where $A$ sends a qubit first instead of $B$ sending a qubit first. The only thing $E$ cannot "rewind" is the probability of $B$ observing certain outcomes in $\widetilde{\Pi}^{\mathrm{SQKD}}$ - thus the need for her to set the value of $p_{0}$ during device construction.

Theorem 2. Let $\left(U_{F}, U_{R}\right)$ be a collective attack used against $\widetilde{\Pi}^{\mathrm{SQKD}}$ and let $\rho_{A B E}$ be the density operator describing a single iteration of this protocol $\widetilde{\Pi}^{\mathrm{SQKD}}$ when $E$ uses this attack. Then, there exists an attack $\mathcal{E}=\left(p_{0}, U\right)$ against $\Pi^{*}$ such that, if $\sigma_{A B E}$ is the resulting density operator when running $\Pi^{*}$ using attack $\mathcal{E}$, it holds that $\rho_{A B E}=\sigma_{A B E}$ assuming the probability that $B$ chooses Measure and Resend in $\widetilde{\Pi}^{\mathrm{SQKD}}$ is the same as in $\Pi^{*}$ (and thus the probability of choosing Reflect is also the same in both protocols).

Proof. Let $\left(U_{F}, U_{R}\right)$ be a collective attack against $\widetilde{\Pi}^{\mathrm{S} Q \mathrm{KD}}$. Since Theorem 1 applies, there exists an equivalent restricted attack consisting of the forward operator $\mathcal{F}$ as described in Equation 3 . We construct the desired attack $\mathcal{E}$ against $\Pi^{*}$.

Consider the following operator $\mathrm{Rw}$ to be used against $\Pi^{*}$ in order to "rewind" the forward channel attack. The action of this operator is:

$$
\begin{aligned}
\mathbf{R w}|00\rangle_{A_{1} A_{2}} & =\frac{q_{0}|000\rangle_{A_{1} A_{2} E}+\sqrt{1-q_{1}^{2}}|10 f\rangle_{A_{1} A_{2} E}}{\sqrt{1-q_{1}^{2}+q_{0}^{2}}} \\
\mathbf{R w}|11\rangle_{A_{1} A_{2}} & =\frac{\sqrt{1-q_{0}^{2}}|01 e\rangle_{A_{1} A_{2} E}+q_{1}|110\rangle_{A_{1} A_{2} E}}{\sqrt{1-q_{0}^{2}+q_{1}^{2}}},
\end{aligned}
$$

where $|e\rangle$ and $|f\rangle$ are the states resulting from the application of $\mathcal{F}$ (see Equations 4 and 5). We claim that Rw is an isometry (and thus can be extended to a unitary operation). This is not difficult to see: let $\left|v_{0}\right\rangle=\mathbf{R w}|00\rangle$ and $\left|v_{1}\right\rangle=\mathbf{R} \mathbf{w}|11\rangle$. It is clear that $\left\langle v_{0} \mid v_{1}\right\rangle=\left\langle v_{1} \mid v_{0}\right\rangle=0$. Furthermore:

$$
\left\langle v_{0} \mid v_{0}\right\rangle=\frac{1}{1-q_{1}^{2}+q_{0}^{2}}\left(q_{0}^{2}+1-q_{1}^{2}\right)=1,
$$

and similarly $\left\langle v_{1} \mid v_{1}\right\rangle=1$. Thus, Rw is an isometry from $\mathcal{H}_{A_{1} A_{2}} \rightarrow \mathcal{H}_{A_{1} A_{2} E}$. We abuse notation from here and assume Rw is a unitary operator (its action on other states may be arbitrary).

Now, consider the following attack against $\Pi^{*}: \mathcal{E}=$ $\left(p_{0},\left(I_{A_{1}} \otimes U_{R}\right) \cdot \mathbf{R w}\right)$, where $p_{0}=\frac{1}{2}\left(1-q_{1}^{2}+q_{0}^{2}\right)$ (and, so, $\left.p_{1}=1-p_{0}=\frac{1}{2}\left(1-q_{0}^{2}+q_{1}^{2}\right)\right)$. We claim this is the desired 
attack. Indeed, if $B$ chooses to Reflect in $\widetilde{\Pi}^{\mathrm{SQKD}}$, then the state of the system after the qubit leaves $B$ 's lab, but before $E$ applies $U_{R}$ is:

$$
\begin{aligned}
\left|\psi_{A_{1} A_{2} B E}^{R}\right\rangle & =\frac{1}{\sqrt{2}}\left(q_{0}|0000\rangle+\sqrt{1-q_{0}^{2}}|010 e\rangle\right) \\
& +\frac{1}{\sqrt{2}}\left(\sqrt{1-q_{1}^{2}}|100 f\rangle+q_{1}|1100\rangle\right),
\end{aligned}
$$

where the order of the systems on the right-hand-side of the above expression are: $A_{1} A_{2} B E$ (note $B$ is un-entangled from the above system). At this point, $E$ has control only of the $A_{2} E$ subspace.

On the other hand, if $B$ chooses Reflect in $\Pi^{*}$, then the state of the system after $E$ applies Rw but before finishing the attack with $U_{R}$, is:

$$
\begin{aligned}
& \left|\phi_{A_{1} A_{2} B E}^{R}\right\rangle= \\
& \frac{\sqrt{p_{0}}}{\sqrt{1-q_{1}^{2}+q_{0}^{2}}}\left(q_{0}|0000\rangle+\sqrt{1-q_{1}^{2}}|100 f\rangle\right) \\
+ & \frac{\sqrt{p_{1}}}{\sqrt{1-q_{0}^{2}+q_{1}^{2}}}\left(\sqrt{1-q_{0}^{2}}|010 e\rangle+q_{1}|1100\rangle\right) \\
= & \frac{1}{\sqrt{2}}\left(q_{0}|0000\rangle+\sqrt{1-q_{1}^{2}}|100 f\rangle\right) \\
+ & \frac{1}{\sqrt{2}}\left(\sqrt{1-q_{0}^{2}}|010 e\rangle+q_{1}|1100\rangle\right),
\end{aligned}
$$

from which it is clear that $\left|\psi_{A_{1} A_{2} B E}^{R}\right\rangle \equiv\left|\phi_{A_{1} A_{2} B E}^{R}\right\rangle$. With $\widetilde{\Pi}^{\mathrm{SQKD}}, E$ will then apply $U_{R}$ (acting on $A_{2} E$ ) to $\left|\psi^{R}\right\rangle$ and then forward the $A_{2}$ system to Alice; with $\Pi^{*}, E$ will apply the same $U_{R}$ (also acting on the subspace $A_{2} E$ ) to $\left|\phi^{R}\right\rangle$ and forwards both $A_{1}$ and $A_{2}$ to Alice. Regardless, we find the quantum system held by all three parties to be equal.

The case when $B$ chooses to Measure and Resend is similar. Indeed, in this case, consider the state before $E$ applies $U_{R}$ for $\widetilde{\Pi}^{\mathrm{SQKD}}$ :

$$
\begin{aligned}
\left|\psi_{A_{1} A_{2} B E}^{M}\right\rangle & =\frac{1}{\sqrt{2}}\left(q_{0}|0000\rangle+\sqrt{1-q_{0}^{2}}|011 e\rangle\right) \\
& +\frac{1}{\sqrt{2}}\left(\sqrt{1-q_{1}^{2}}|100 f\rangle+q_{1}|1110\rangle\right) .
\end{aligned}
$$

And, the case for $\Pi^{*}$ after $E$ applies Rw but before $U_{R}$ is:

$$
\begin{aligned}
& \left|\phi_{A_{1} A_{2} B E}^{M}\right\rangle= \\
& \frac{\sqrt{p_{0}}}{\sqrt{1-q_{1}^{2}+q_{0}^{2}}}\left(q_{0}|0000\rangle+\sqrt{1-q_{1}^{2}}|100 f\rangle\right) \\
+ & \frac{\sqrt{p_{1}}}{\sqrt{1-q_{0}^{2}+q_{1}^{2}}}\left(\sqrt{1-q_{0}^{2}}|011 e\rangle+q_{1}|1110\rangle\right) \\
= & \frac{1}{\sqrt{2}}\left(q_{0}|0000\rangle+\sqrt{1-q_{1}^{2}}|100 f\rangle\right) \\
+ & \frac{1}{\sqrt{2}}\left(\sqrt{1-q_{0}^{2}}|011 e\rangle+q_{1}|1110\rangle\right) .
\end{aligned}
$$

Again, we conclude the two systems will be the same after application of $U_{R}$. The final density operator will then be a mixture of the two pure states. Assuming the probability that $B$ chooses to Measure and Resend in $\Pi^{\mathrm{SQKD}}$ is equal to the probability he chooses this option in $\Pi^{*}$ (and thus, the probability of choosing Reflect is also equal in both protocols) the resulting density operators will be identical.

Notice that, when attacking $\Pi^{*}, E$ has control of both $A_{1}$ and $A_{2}$ (which she does not when attacking $\widetilde{\Pi}^{\mathrm{S} Q K D}$ ). Thus, she has possibly more attack strategies against $\Pi^{*}$. However, for every attack against $\widetilde{\Pi}^{\mathrm{SQKD}}$, there exists an equivalent attack against $\Pi^{*}$. Thus, to prove security of $\widetilde{\Pi}^{\mathrm{SQKD}}$ (and thus $\Pi^{\mathrm{SQKD}}$, our goal), it suffices to analyze $\Pi^{*}$ as $E$ has potentially more attack capabilities against the latter. Indeed, we have the following "chain:"

$$
\Pi^{*} \Longrightarrow \Pi_{r e s}^{*} \Longrightarrow \widetilde{\Pi}^{\mathrm{SQKD}} \Longrightarrow \Pi^{\mathrm{SQKD}},
$$

where $\Pi_{\text {res }}^{*}$ is the protocol $\Pi^{*}$ but with $E$ restricted to attacks of the form $\mathcal{E}=\left(p_{0},\left(I_{A_{1}} \otimes U\right) \cdot \mathbf{R w}\right)$.

\section{Final Key-Rate Bound}

Consider the protocol $\Pi^{*}$ introduced in the previous subsection. There are two "modes" to it: either $B$ chooses to Measure and Resend (with probability $P_{M}$ ) or he chooses to Reflect (with probability $P_{R}$ ) - not that these terms have the same meaning as their wording implies. A single iteration of the protocol, then, may be written as the density operator:

$$
\rho_{A_{1} A_{2} B E}=P_{M} \sigma_{A_{1} A_{2} B E}+P_{R} \tau_{A_{1} A_{2} B E},
$$

where:

$$
\sigma_{A_{1} A_{2} B E}=\left[\phi_{\mathbf{A}_{\mathbf{1}} \mathbf{A}_{\mathbf{2}} \mathbf{B E}}^{\mathbf{M}}\right], \quad \tau_{A_{1} A_{2} B E}=\left[\phi_{\mathbf{A}_{\mathbf{1}} \mathbf{A}_{\mathbf{2}} \mathbf{B E}}^{\mathbf{R}}\right]
$$

and $\left|\phi^{M}\right\rangle$ and $\left|\phi^{R}\right\rangle$ are the (pure) states in the event $B$ chooses Measure and Resend or Reflect respectively. Now, only those iterations where $B$ chooses Measure and Resend are used for key distillation and, so, to compute the key-rate of this protocol, we need to compute $S\left(A_{1}^{Z} \mid E\right)_{\sigma}$ where we use $A_{1}^{Z}$ to denote the result of $A$ measuring her $A_{1}$ register in the $Z$ basis (recall $A$ uses only $A_{1}$ for her key distillation). However, we will first analyze $S\left(A_{1} \mid E\right)_{\tau}$ and use this to bound the entropy in $\sigma$.

It was shown in [24], that for any density operator acting on a tripartite Hilbert space $\mathcal{H}_{A} \otimes \mathcal{H}_{B} \otimes \mathcal{H}_{E}$, that if $A$ and $B$ make measurements in the $Z$ or $X$ basis, then:

$$
S\left(A^{Z} \mid E\right)+S\left(A^{X} \mid B\right) \geq 1,
$$

where we use $A^{Z}$ (respectively $A^{X}$ ) to denote the register storing the result of a $Z$ (respectively $X$ ) basis measurement on the $A$ system. Using this, we may easily prove the following:

Lemma 1. Let $\tau_{A_{1} A_{2} B E}$ be the state of the system if $B$ chooses Reflect in protocol $\Pi^{*}$ and let $Q_{X}$ be the error rate in the $X$ basis between registers $A_{1}$ and $A_{2}$. Then:

$$
S\left(A_{1}^{Z} \mid E\right)_{\tau} \geq 1-h\left(Q_{X}\right) .
$$


Proof. Note that $B$ is completely independent of the $A_{1} A_{2} E$ system; i.e., $\tau_{A_{1} A_{2} B E} \equiv \tau_{A_{1} A_{2} E} \otimes[\mathbf{0}]_{B}$. Thus, we may simply consider the state resulting from tracing out $B$ which acts on the tripartite system $\mathcal{H}_{A_{1}} \otimes \mathcal{H}_{A_{2}} \otimes \mathcal{H}_{E}$. Using the uncertainty relation described above (see Equation 12, replacing $B$ with $A_{2}$ (thus, in a way, we are imagining $A$ as two people - one who holds the $A_{1}$ register and the other who holds the $A_{2}$ register - of course in real life they are one individual), we have:

$$
\begin{aligned}
S\left(A_{1}^{Z} \mid E\right) & \geq 1-S\left(A_{1}^{X} \mid A_{2}\right) \\
& \geq 1-H\left(A_{1}^{X} \mid A_{2}^{X}\right) \\
& =1-h\left(Q_{X}\right),
\end{aligned}
$$

where the last inequality follows from the fact that measurements can only increase entropy.

We will use the conditional entropy of $\tau_{A_{1} E}$ to compute a bound on the entropy in $\sigma_{A_{1} E}$, thus giving us our desired keyrate computation. For the following result, we will assume a symmetric attack in that the observed $Z$ basis noise is equal in both forward and reverse channels; this is only to make the algebra more amicable - analyzing an asymmetric channel would follow the same process, just with slightly more, yet trivial, algebra.

Before we continue, however, we require one lemma which, though straight-forward to show, we include for completeness:

Lemma 2. Let $\sigma=\left|v_{0}\right\rangle\left\langle v_{1}|+| v_{1}\right\rangle\left\langle v_{0}\right|$. Then:

$$
\|\sigma\| \leq 2 \sqrt{\left\langle v_{0} \mid v_{0}\right\rangle\left\langle v_{1} \mid v_{1}\right\rangle}
$$

Proof. Recall that the trace norm is invariant to unitary changes in basis. We decompose $\left|v_{0}\right\rangle$ and $\left|v_{1}\right\rangle$ as:

$$
\left|v_{0}\right\rangle=x|E\rangle, \quad\left|v_{1}\right\rangle=h e^{i \theta}|E\rangle+d|I\rangle,
$$

where $\langle E \mid E\rangle=\langle I \mid I\rangle=1$ and $\langle E \mid I\rangle=0$. Furthermore, we may assume $x, h, d \in \mathbb{R}$ (any alternative phase of $x$ or $d$ may be absorbed into the corresponding basis vector). In this $\{|E\rangle,|I\rangle\}$ basis, we have:

$$
\sigma=\left(\begin{array}{cc}
x h e^{i \theta}+x h e^{-i \theta} & x d \\
x d & 0
\end{array}\right)=\left(\begin{array}{cc}
2 x R e\left(h e^{i \theta}\right) & x d \\
x d & 0
\end{array}\right),
$$

the eigenvalues of which are:

$$
\lambda_{ \pm}=x \operatorname{Re}\left(h e^{i \theta}\right) \pm \sqrt{x^{2} \operatorname{Re}^{2}\left(h e^{i \theta}\right)+x^{2} d^{2}} .
$$

Writing $\left\langle v_{0} \mid v_{1}\right\rangle=a+b i$, with $a, b \in \mathbb{R}$, we have the following identities (which follows from the decomposition of $\left|v_{0}\right\rangle$ and $\left.\left|v_{1}\right\rangle\right)$ :

$$
\begin{aligned}
& x^{2}=\left\langle v_{0} \mid v_{0}\right\rangle \\
& h e^{i \theta}=\frac{\left\langle v_{0} \mid v_{1}\right\rangle}{x}=\frac{a}{x}+\frac{b}{x} i \Rightarrow h^{2}=\frac{a^{2}}{x^{2}}+\frac{b^{2}}{x^{2}} \\
& h^{2}+d^{2}=\left\langle v_{1} \mid v_{1}\right\rangle \Rightarrow d^{2}=\left\langle v_{1} \mid v_{1}\right\rangle-\frac{a^{2}}{x^{2}}-\frac{b^{2}}{x^{2}} .
\end{aligned}
$$

Substituting these into $\lambda_{ \pm}$yields:

$$
\begin{aligned}
\lambda_{ \pm} & =x \operatorname{Re}\left(h e^{i \theta}\right) \pm \sqrt{x^{2} R e^{2}\left(h e^{i \theta}\right)+x^{2} d^{2}} \\
& =a \pm \sqrt{a^{2}+x^{2}\left\langle v_{1} \mid v_{1}\right\rangle-a^{2}-b^{2}} \\
& =a \pm \sqrt{\left\langle v_{0} \mid v_{0}\right\rangle\left\langle v_{1} \mid v_{1}\right\rangle-b^{2}} .
\end{aligned}
$$

The Cauchy-Schwarz inequality forces $b^{2} \leq\left\langle v_{0} \mid v_{0}\right\rangle\left\langle v_{1} \mid v_{1}\right\rangle$, so the square-root is real. In fact, by the Cauchy-Schwarz inequality:

$$
a^{2}+b^{2} \leq\left\langle v_{0} \mid v_{0}\right\rangle\left\langle v_{1} \mid v_{1}\right\rangle \Rightarrow|a| \leq \sqrt{\left\langle v_{0} \mid v_{0}\right\rangle\left\langle v_{1} \mid v_{1}\right\rangle-b^{2}} .
$$

Now: $\|\sigma\|=\left|\lambda_{+}\right|+\left|\lambda_{-}\right|$. If $a \geq 0$, then, using Equation 17 and letting $\delta=\sqrt{\left\langle v_{0} \mid v_{0}\right\rangle\left\langle v_{1} \mid v_{1}\right\rangle-b^{2}}$ (thus $0 \leq a \leq \delta$ ):

$$
\|\sigma\|=|a+\delta|+|a-\delta|=(a+\delta)+(\delta-a)=2 \delta .
$$

Alternatively, if $a<0$ :

$$
\|\sigma\|=|a+\delta|+|a-\delta|=(a+\delta)+(\delta-a)=2 \delta .
$$

Of course $\delta \leq \sqrt{\left\langle v_{0} \mid v_{0}\right\rangle\left\langle v_{1} \mid v_{1}\right\rangle}$ completing the proof.

Finally, we prove the following theorem which bounds the von Neumann entropy in $\sigma$ allowing us to compute the keyrate of this $\mathrm{SQKD}$ protocol.

Theorem 3. Given $\sigma_{A_{1} A_{2} B E}$ and $\tau_{A_{1} A_{2} B E}$ as defined above in Equation 11, let $Q$ be the error rate in the $Z$ basis observed on a single channel (we assume both channels have the same $Z$ basis error rate). Also, let $\delta$ be defined as:

$$
\delta=2 Q(1-Q)+\left(\frac{1}{2}+2 Q[1-Q]\right) \cdot h\left(\frac{4 Q(1-Q)}{1+4 Q(1-Q)}\right) .
$$

Then, assuming $E$ 's attack is symmetric and of the form $\mathcal{E}=\left(1 / 2,\left(I_{A_{1}} \otimes V\right) \mathbf{R w}\right)$, where $V$ acts on $\mathcal{H}_{A_{2} E}$ and $\mathbf{R w}$ is a "rewind" operator as discussed earlier, it holds that $S\left(A_{1}^{Z} \mid E\right)_{\sigma} \geq f(Q)$, where:

$$
f(Q)= \begin{cases}S\left(A_{1}^{Z} \mid E\right)_{\tau}-\delta & \text { if } S\left(A_{1}^{Z} \mid E\right)_{\tau} \geq 2 \delta \\ \frac{1}{2} S\left(A_{1}^{Z} \mid E\right)_{\tau} & \text { otherwise }\end{cases}
$$

Proof. Let $U$ be an arbitrary attack operator used against $\Pi^{*}$ (this is an isometry from $\mathcal{H}_{A_{1} A_{2}}$ to $\mathcal{H}_{A_{1} A_{2} E}$ ). Also, let $\left|\phi_{ \pm}\right\rangle=$ $\frac{1}{\sqrt{2}}(|00\rangle \pm|11\rangle)$ be two Bell states. Without loss of generality, we may write $U$ 's action as:

$$
\begin{aligned}
U\left|\phi_{+}\right\rangle & =\sum_{a, b \in\{0,1\}}|a, b\rangle_{A_{1} A_{2}} \otimes\left|e_{a b}\right\rangle_{E} \\
U\left|\phi_{-}\right\rangle & =\sum_{a, b \in\{0,1\}}|a, b\rangle_{A_{1} A_{2}} \otimes\left|f_{a b}\right\rangle_{E},
\end{aligned}
$$

where the states $\left|e_{a b}\right\rangle$ and $\left|f_{a b}\right\rangle$ are arbitrary, not necessarily normalized nor orthogonal, states in $\mathcal{H}_{E}$. The density operator $\tau_{A_{1}^{Z} A_{2}^{Z} E}$ (which is the state of the system when $B$ chooses Reflect in protocol $\Pi^{*}$ and $A$ measures both her qubits in 
the $Z$ basis - an operation denoted by $\mathcal{M}$ below) when faced with this attack is found to be:

$$
\begin{aligned}
& \tau_{A_{1}^{Z} A_{2}^{Z} E}=\mathcal{M}\left[U\left[\phi_{+}\right] U^{*}\right]=\sum_{a, b}[\mathbf{a}, \mathbf{b}] \otimes\left[\mathbf{e}_{\mathbf{a b}}\right] \\
\Rightarrow & \tau_{A_{1}^{Z} E}=\sum_{a \in\{0,1\}}[\mathbf{a}] \otimes\left(\left[\mathbf{e}_{\mathbf{a} 0}\right]+\left[\mathbf{e}_{\mathbf{a} 1}\right]\right) .
\end{aligned}
$$

Likewise, we may compute $\sigma$, the density operator for those iterations where $B$ chooses Measure and Resend (note that, below, we define $\left.P(z)=z z^{*}\right)$ :

$$
\begin{aligned}
& \left.\sigma_{A_{1}^{Z} A_{2}^{Z} E}=\mathcal{M}\left[U\left(\frac{1}{2}[\mathbf{0 0}]+\frac{1}{2}[\mathbf{1 1}]\right)\right) U^{*}\right] \\
& =\mathcal{M}\left[\frac{1}{4} P\left(U\left|\phi_{+}\right\rangle+U\left|\phi_{-}\right\rangle\right)+\frac{1}{4} P\left(U\left|\phi_{+}\right\rangle-U\left|\phi_{-}\right\rangle\right)\right] \\
& =\frac{1}{4} \sum_{a, b}[\mathbf{a}, \mathbf{b}] \otimes\left(P\left[\left|e_{a, b}\right\rangle+\left|f_{a, b}\right\rangle\right]+P\left[\left|e_{a, b}\right\rangle-\left|f_{a, b}\right\rangle\right]\right) \\
& =\frac{1}{2} \sum_{a, b}[\mathbf{a}, \mathbf{b}] \otimes\left(\left[\mathbf{e}_{\mathbf{a}, \mathbf{b}}\right]+\left[\mathbf{f}_{\mathbf{a}, \mathbf{b}}\right]\right) .
\end{aligned}
$$

From the above, it is not difficult to see:

$$
\sigma_{A_{1}^{Z} E}=\frac{1}{2} \tau_{A_{1}^{Z} E}+\frac{1}{2} \mu_{A_{1}^{Z} E}
$$

where:

$$
\mu_{A_{1}^{Z} E}=\sum_{a}[\mathbf{a}] \otimes\left(\left[\mathbf{f}_{\mathbf{a} 0}\right]+\left[\mathbf{f}_{\mathbf{a} \mathbf{1}}\right]\right)
$$

Thus, by concavity of conditional entropy, we have:

$$
S\left(A_{1}^{Z} \mid E\right)_{\sigma} \geq \frac{1}{2} S\left(A_{1}^{Z} \mid E\right)_{\tau}+\frac{1}{2} S\left(A_{1}^{Z} \mid E\right)_{\mu}
$$

Recall that we are actually interested in proving security against $\widetilde{\Pi}^{\mathrm{S} Q K D}$ and so only need to concern ourselves with attacks of the form: $U=\left(I_{A_{1}} \otimes V\right)$ Rw. From Equation 8 and noting that, since we are assuming a symmetric attack in that $p_{0}=p_{1}=1 / 2$ and so $q_{0}^{2}=q_{1}^{2}$, the action of $\mathbf{R w}$ on $\left|\phi_{ \pm}\right\rangle$is:

$$
\mathbf{R} \mathbf{w}\left|\phi_{ \pm}\right\rangle=\frac{1}{\sqrt{2}}\left(q_{0}|000\rangle+\overline{q_{1}}|10 f\rangle \pm \overline{q_{0}}|01 e\rangle \pm q_{1}|110\rangle\right),
$$

where we use $\overline{q_{0}}$ to mean $\sqrt{1-q_{0}^{2}}$ (and similarly for $\overline{q_{1}}$ ) and where $|e\rangle$ and $|f\rangle$ are defined in Equations 4 and 5

Now, we may, without loss of generality, describe $V$ 's action as follows (recall $V$ is a unitary operator acting on $\mathcal{H}_{A_{2} E}$ ):

$$
\begin{aligned}
V|00\rangle & =\left|0, e_{0}\right\rangle+\left|1, e_{1}\right\rangle, & V|10\rangle & =\left|0, e_{2}\right\rangle+\left|1, e_{3}\right\rangle \\
V|0 f\rangle & =\left|0, f_{0}\right\rangle+\left|1, f_{1}\right\rangle, & V|1 e\rangle & =\left|0, f_{2}\right\rangle+\left|1, f_{3}\right\rangle
\end{aligned}
$$

Thus, after applying attack $U=\left(I_{A_{1}} \otimes V\right) \cdot \mathbf{R w}$ to $\left|\phi_{ \pm}\right\rangle$, we find:

$$
\begin{aligned}
\sqrt{2} U\left|\phi_{ \pm}\right\rangle & =q_{0}\left|00 e_{0}\right\rangle+q_{0}\left|01 e_{1}\right\rangle+\overline{q_{1}}\left|10 f_{0}\right\rangle+\overline{q_{1}}\left|11 f_{1}\right\rangle \\
& \pm \overline{q_{0}}\left|00 f_{2}\right\rangle \pm \overline{q_{0}}\left|01 f_{3}\right\rangle \pm q_{1}\left|10 e_{2}\right\rangle \pm q_{1}\left|11 e_{3}\right\rangle
\end{aligned}
$$

Translating to notation used in Equation 19, we find:

$$
\begin{aligned}
\left|e_{00}\right\rangle & =\frac{1}{\sqrt{2}}\left(q_{0}\left|e_{0}\right\rangle+\overline{q_{0}}\left|f_{2}\right\rangle\right) \\
\left|e_{01}\right\rangle & =\frac{1}{\sqrt{2}}\left(q_{0}\left|e_{1}\right\rangle+\overline{q_{0}}\left|f_{3}\right\rangle\right) \\
\left|e_{10}\right\rangle & =\frac{1}{\sqrt{2}}\left(\overline{q_{1}}\left|f_{0}\right\rangle+q_{1}\left|e_{2}\right\rangle\right) \\
\left|e_{11}\right\rangle & =\frac{1}{\sqrt{2}}\left(\overline{q_{1}}\left|f_{1}\right\rangle+q_{1}\left|e_{3}\right\rangle\right),
\end{aligned}
$$

while the states $\left|f_{a b}\right\rangle$ are found simply by taking $\left|e_{a b}\right\rangle$ and changing the "+" to a "-" in between the two kets.

Our goal is to determine a bound on $S\left(A_{1}^{Z} \mid E\right)_{\mu}$ using $S\left(A_{1}^{Z} \mid E\right)_{\tau}$. To do so, we will use a continuity bound on conditional entropy determined by Winter in [25] (a tighter version of the Alicki-Fannes inequality [26]). Using this bound, we have:

$$
\left|S\left(A_{1}^{Z} \mid E\right)_{\tau}-S\left(A_{1}^{Z} \mid E\right)_{\mu}\right| \leq \epsilon+(1+\epsilon) h\left(\frac{\epsilon}{1+\epsilon}\right),
$$

where:

$$
\frac{1}{2}\left\|\tau_{A_{1}^{Z} E}-\mu_{A_{1}^{Z} E}\right\| \leq \epsilon \leq 1 .
$$

Thus, if we bound the trace distance between $\tau$ and $\mu$ we can determine an upper-bound on $S\left(A_{1}^{Z} \mid E\right)_{\mu}$ thus giving us our result.

By the triangle inequality, we have:

$$
\begin{aligned}
\left\|\tau_{A_{1}^{Z} E}-\mu_{A_{1}^{Z} E}\right\| & \leq \sum_{a, b}\left\|\left[\mathbf{e}_{\mathbf{a b}}\right]-\left[\mathbf{f}_{\mathbf{a b}}\right]\right\| \\
& =q_{0} \overline{q_{0}} \|\left|e_{0}\right\rangle\left\langle f_{2}|+| f_{2}\right\rangle\left\langle e_{0} \|\right| \\
& +q_{0} \overline{q_{0}}||\left|e_{1}\right\rangle\left\langle f_{3}|+| f_{3}\right\rangle\left\langle e_{1} \|\right| \\
& +\overline{q_{1}} q_{1}\left|\| f_{0}\right\rangle\left\langle e_{2}|+| e_{2}\right\rangle\left\langle f_{0} \|\right| \\
& +\overline{q_{1}} q_{1}||\left|f_{1}\right\rangle\left\langle e_{3}|+| e_{3}\right\rangle\left\langle f_{1} \|\right| \\
& \leq 2 q_{0} \overline{q_{0}} \sqrt{\left\langle e_{0} \mid e_{0}\right\rangle\left\langle f_{2} \mid f_{2}\right\rangle} \\
& +2 q_{0} \overline{q_{0}} \sqrt{\left\langle e_{1} \mid e_{1}\right\rangle\left\langle f_{3} \mid f_{3}\right\rangle} \\
& +2 \overline{q_{1}} q_{1} \sqrt{\left\langle e_{2} \mid e_{2}\right\rangle\left\langle f_{0} \mid f_{0}\right\rangle} \\
& +2 \overline{q_{1}} q_{1} \sqrt{\left\langle e_{3} \mid e_{3}\right\rangle\left\langle f_{1} \mid f_{1}\right\rangle},
\end{aligned}
$$

where the last inequality follows from Lemma 2 .

Assuming the attack is symmetric, with $Q$ being the $Z$ basis noise in each channel (i.e., the probability of a $|i\rangle$ flipping to a $|1-i\rangle$ in either channel is $Q$ ), then it is easy to see that $q_{0}=q_{1}=\sqrt{1-Q}$ (thus $\overline{q_{1}}=\overline{q_{0}}=\sqrt{Q}$ ) and also:

$$
\begin{aligned}
& \left\langle e_{0} \mid e_{0}\right\rangle=\left\langle e_{3} \mid e_{3}\right\rangle=\left\langle f_{0} \mid f_{0}\right\rangle=\left\langle f_{3} \mid f_{3}\right\rangle=(1-Q) \\
& \left\langle e_{1} \mid e_{1}\right\rangle=\left\langle e_{2} \mid e_{2}\right\rangle=\left\langle f_{1} \mid f_{1}\right\rangle=\left\langle f_{2} \mid f_{2}\right\rangle=Q
\end{aligned}
$$

(Note that these values are observable by $A$ and $B$ in both $\Pi^{*}$ and $\Pi^{\mathrm{S} Q K D}$.)

Thus:

$$
\begin{aligned}
\frac{1}{2}\left\|\tau_{A_{1}^{Z} E}-\mu_{A_{1}^{Z} E}\right\| & \leq \frac{1}{2}(8 \sqrt{Q(1-Q)} \sqrt{Q(1-Q)}) \\
& =4 Q(1-Q) .
\end{aligned}
$$


From Equation 23, and setting $\epsilon=4 Q(1-Q)$, we have:

$$
S\left(A_{1}^{Z} \mid E\right)_{\mu} \geq S\left(A_{1}^{Z} \mid E\right)_{\tau}-\epsilon-(1+\epsilon) h\left(\frac{\epsilon}{1+\epsilon}\right) .
$$

Combining this with Equation 22, and also noting that, since $\mu_{A_{1}^{Z} E}$ is a classical-quantum state, and so $S\left(A_{1}^{Z} \mid E\right)_{\mu} \geq 0$, completes the proof.

Note that the only place we used a symmetric assumption was in the computation of $q_{i},\left\langle e_{i} \mid e_{i}\right\rangle$, and $\left\langle f_{i} \mid f_{i}\right\rangle$ and in the assumption that $B$ sends Bell states $\left|\phi_{ \pm}\right\rangle$; if the attack was asymmetric, the only thing that would change would be these quantities - however our technique used in the proof of Theorem 3 would still apply, it would just require more algebra. Note, however, that since these are observable quantities, $A$ and $B$ can even enforce that $E$ use a symmetric attack.

The key-rate of the protocol $\Pi_{\text {res }}^{*}$, therefore, is:

$$
\begin{aligned}
r & =S\left(A_{1}^{Z} \mid E\right)_{\sigma}-H\left(A_{1}^{Z} \mid B^{Z}\right)_{\sigma} \\
& \geq g\left(Q, Q_{X}\right)-h(Q),
\end{aligned}
$$

where:

$$
g\left(Q, Q_{X}\right)= \begin{cases}1-h\left(Q_{X}\right)-\delta & \text { if } 1-h\left(Q_{X}\right) \geq 2 \delta \\ \frac{1}{2}\left(1-h\left(Q_{X}\right)\right) & \text { otherwise }\end{cases}
$$

and $\delta$ is defined in Theorem 3 . Note that, above, we used the trivial fact that $H\left(A_{1}^{Z} \mid B^{Z}\right)_{\sigma}=h(Q)$ in this scenario (an asymmetric attack will be different, but also easily computed). Of course, by our analysis conducted above, the key-rate of $\Pi^{\mathrm{SQKD}}$ can only be higher than this.

We now evaluate our new key-rate bound, in particular its noise tolerance, comparing to current known results. In [14], a lower bound on the key-rate of $\Pi^{\mathrm{SQKD}}$ was derived without mismatched measurements. In [17], we derived a new bound, but using numerous mismatched measurement statistics (e.g., the probability of a $|+\rangle$ being measured as a $|0\rangle$ ). Such statistics give greater information on the attack being used (thus increasing the bound on $S(A \mid E)$ ) but at the cost of wasting quantum communication on error estimation.

As shown in Table [1] the noise tolerance of our new bound here is higher than previous best work utilizing only noise statistics (and not mismatched measurements) [14]. However, it is lower than the bound derived using mismatched measurements as we did in [17]. Note that we did not require any mismatched measurements for our derivation in this paper, thus it is expected that our key-rate bound may not be as high as that discovered in [17]. In that work, we used over 12 different measurement statistics to bound $S(A \mid E)$. In this security proof, we are only using three measurement statistics: the error rate in the $Z$ basis (for both channels) and the $X$ error rate observed by $A$. Thus, it is not surprising our noise tolerance here is lower. An interesting open question is whether this difference is primarily an artifact of our proof (in particular, Theorem 3 may not be tight) or whether this shows the necessity of using mismatched statistics for this protocol (such statistics are known to be highly beneficial to limitedresource protocols [27] and even required for some SQKD

\begin{tabular}{l|c|c|c} 
& Old Proof [14] & New Proof & w/MM [17] \\
\hline$Q_{X}=Q$ & $5.34 \%$ & $\mathbf{6 . 1 4} \%$ & $11 \%$ \\
$Q_{X}=2 Q(1-Q)$ & $4.57 \%$ & $\mathbf{4 . 8 2} \%$ & $7.9 \%$ \\
$Q_{X}=\frac{1}{2} Q$ & $5.92 \%$ & $\mathbf{7 . 5} \%$ & $15.12 \%$
\end{tabular}

SHOWING THE MAXIMAL NOISE TOLERANCE (I.E., THE MAXIMUM $Q$ FOR WHICH THE KEY-RATE OF $\Pi^{\text {SQKD }}$ REMAINS POSITIVE) OF $\Pi^{\text {SQKD }}$ DERIVED HERE (MIDDLE COLUMN LABELED "NEW PROOF”). ALSO COMPARING WITH CURRENT KNOWN RESULTS FOR THIS PROTOCOL. "OLD PROOF" IS FROM 14 AND BOUNDED THE KEY-RATE USING ONLY THREE NOISE STATISTICS (AS WAS DONE HERE). "W/MM" WAS FROM [17] AND UTILIZED MISMATCHED MEASUREMENTS (IN TOTAL OVER 12 DIFFERENT MEASUREMENT STATISTICS AS OPPOSED TO ONLY THREE USED HERE). OUR NEW RESULT IS HIGHER THAN THAT IN [14], BUT NOT AS HIGH AS WHEN UTILIZING MISMATCHED MEASUREMENT STATISTICS. SEE TEXT FOR FURTHER EXPLANATION.

protocols [28]). We intend to investigate this further in the near future.

\section{Comment on General Attacks}

Normally, one may extend the computations done for collective attacks to produce security against general attacks, in the asymptotic scenario, by invoking a de Finetti type argument [29], [30], [31]. It seems like this should also hold for our security proof here, however, due to our reliance on the restricted collective attack, this would require a more rigorous proof 1 . We suspect the results in this paper would carry through to imply security against general attacks, however we leave a formal, rigorous, proof of this as future work.

\section{Closing Remarks}

In this paper, we presented an entirely new proof of security for a semi-quantum protocol. By first reducing the problem to an equivalent entanglement-based protocol and then using a quantum entropic uncertainty relation, along with a continuity bound on conditional von Neumann entropy, we were able to derive a cleaner key-rate expression for this protocol than previous work. Furthermore, our new keyrate has a higher noise tolerance than previous work without mismatched measurements. Of course, our new key-rate has a lower tolerance than when using mismatched measurements. An open question worth investigating is whether this is an artifact of our proof technique or if mismatched measurements are absolutely required for this protocol to achieve optimal noise tolerance.

This new technique we developed in this paper, along with the various security results produced along the way, such as the restricted attack, may hold great application in studying other protocols relying on a two-way channel. It may also hold the key to studying SQKD protocols relying on higherdimensional quantum channels such as the one proposed in [20] based on quantum walks. We suspect this technique can be suitably adapted to handle other two-way protocols and other semi-quantum protocols in higher dimensions.

\footnotetext{
${ }^{1}$ Thanks to Rotem Liss for our conversations on this.
} 


\section{REFERENCES}

[1] Valerio Scarani, Helle Bechmann-Pasquinucci, Nicolas J. Cerf, Miloslav Dušek, Norbert Lütkenhaus, and Momtchil Peev. The security of practical quantum key distribution. Rev. Mod. Phys., 81:1301-1350, Sep 2009.

[2] Michel Boyer, Dan Kenigsberg, and Tal Mor. Quantum key distribution with classical bob. Phys. Rev. Lett., 99:140501, Oct 2007.

[3] Michel Boyer, Ran Gelles, Dan Kenigsberg, and Tal Mor. Semiquantum key distribution. Phys. Rev. A, 79:032341, Mar 2009.

[4] Xiangfu Zou, Daowen Qiu, Lvzhou Li, Lihua Wu, and Lvjun Li. Semiquantum-key distribution using less than four quantum states. Phys. Rev. A, 79:052312, May 2009.

[5] Walter O Krawec. Restricted attacks on semi-quantum key distribution protocols. Quantum Information Processing, 13(11):2417-2436, 2014.

[6] Walter O Krawec. Mediated semiquantum key distribution. Physical Review A, 91(3):032323, 2015.

[7] Wang Jian, Zhang Sheng, Zhang Quan, and Tang Chao-Jing. Semiquantum key distribution using entangled states. Chinese Physics Letters, 28(10):100301, 2011.

[8] Hua Lu and Qing-Yu Cai. Quantum key distribution with classical alice. International Journal of Quantum Information, 6(06):1195-1202, 2008.

[9] Kun-Fei Yu, Chun-Wei Yang, Ci-Hong Liao, and Tzonelih Hwang. Authenticated semi-quantum key distribution protocol using bell states. Quantum Information Processing, 13(6):1457-1465, 2014.

[10] Xiangfu Zou, Daowen Qiu, Shengyu Zhang, and Paulo Mateus. Semiquantum key distribution without invoking the classical partys measurement capability. Quantum Information Processing, pages 1-16, 2015.

[11] Qin Li, Wai Hong Chan, and Shengyu Zhang. Real semiquantum key distribution with secure delegated quantum computation. arXiv preprint arXiv:1508.07090 2015.

[12] Michel Boyer, Matty Katz, Rotem Liss, and Tal Mor. Experimentally feasible protocol for semiquantum key distribution. Physical Review A 96(6):062335, 2017.

[13] Takayuki Miyadera. Relation between information and disturbance in quantum key distribution protocol with classical alice. Int. J. of Quantum Information, 9, 2011.

[14] Walter O Krawec. Security proof of a semi-quantum key distribution protocol. In Information Theory (ISIT), 2015 IEEE International Symposium on, pages 686-690. IEEE, 2015.

[15] Walter O Krawec. Security of a semi-quantum protocol where reflections contribute to the secret key. Quantum Information Processing, 15(5):2067-2090, 2016.

[16] Wei Zhang, Daowen Qiu, Xiangfu Zou, and Paulo Mateus. A singlestate semi-quantum key distribution protocol and its security proof. arXiv preprint arXiv:1612.03087. 2016.

[17] Walter O. Krawec. Quantum key distribution with mismatched measurements over arbitrary channels. Quantum Information and Computation, 17(3 and 4):209-241, 2017

[18] Stephen M Barnett, Bruno Huttner, and Simon JD Phoenix. Eavesdropping strategies and rejected-data protocols in quantum cryptography. Journal of Modern Optics, 40(12):2501-2513, 1993.

[19] Shun Watanabe, Ryutaroh Matsumoto, and Tomohiko Uyematsu. Tomography increases key rates of quantum-key-distribution protocols. Physical Review A, 78(4):042316, 2008.

[20] Chrysoula Vlachou, Walter Krawec, Paulo Mateus, Nikola Paunkovic, and Andre Souto. Quantum key distribution with quantum walks. arXiv preprint arXiv:1710.07979. 2017.

[21] Normand J Beaudry, Marco Lucamarini, Stefano Mancini, and Renato Renner. Security of two-way quantum key distribution. Physical Review A, 88(6):062302, 2013.

[22] M.A. Nielsen and I.L. Chuang. Quantum Computation and Quantum Information. Cambridge University Press, Cambridge, MA, 2000.

[23] Igor Devetak and Andreas Winter. Distillation of secret key and entanglement from quantum states. Proceedings of the Royal Society A: Mathematical, Physical and Engineering Science, 461(2053):207-235, 2005.

[24] Mario Berta, Matthias Christandl, Roger Colbeck, Joseph M Renes, and Renato Renner. The uncertainty principle in the presence of quantum memory. Nature Physics, 6(9):659-662, 2010.

[25] Andreas Winter. Tight uniform continuity bounds for quantum entropies: conditional entropy, relative entropy distance and energy constraints. Communications in Mathematical Physics, 347(1):291-313, 2016.
[26] Robert Alicki and Mark Fannes. Continuity of quantum conditional information. Journal of Physics A: Mathematical and General, 37(5):L55, 2004.

[27] Kiyoshi Tamaki, Marcos Curty, Go Kato, Hoi-Kwong Lo, and Koji Azuma. Loss-tolerant quantum cryptography with imperfect sources. Physical Review A, 90(5):052314, 2014.

[28] Walter O Krawec and Eric P Geiss. Limited resource semi-quantum key distribution. arXiv preprint arXiv:1710.05076. 2017.

[29] Renato Renner, Nicolas Gisin, and Barbara Kraus. Information-theoretic security proof for quantum-key-distribution protocols. Phys. Rev. A, 72:012332, Jul 2005.

[30] Matthias Christandl, Robert Konig, and Renato Renner. Postselection technique for quantum channels with applications to quantum cryptography. Phys. Rev. Lett., 102:020504, Jan 2009.

[31] Renato Renner. Symmetry of large physical systems implies independence of subsystems. Nature Physics, 3(9):645-649, 2007. 